\title{
The Use of Gadolinium in Musculoskeletal MRI-Time to Rethink?
}

\author{
Arwa Elawad ${ }^{1}$ Amit Shah ${ }^{1}$ Mark Davies ${ }^{2}$ Rajesh Botchu ${ }^{2}$ \\ ${ }^{1}$ Department of Radiology, University Hospitals of Leicester, \\ Leicester, United Kingdom \\ 2 Department of Radiology, Royal Orthopaedic Hospital, Birmingham, \\ United Kingdom \\ Address for correspondence Rajesh Botchu, FRCR, Department of \\ Radiology, Royal Orthopaedic Hospital, Birmingham B31 2AP, United \\ Kingdom (e-mail: drrajeshb@gmail.com). \\ Indian J Radiol Imaging 2021;31:635-643.
}

\begin{abstract}
Keywords

- gadolinium

- contrast

- musculoskeletal

- magnetic resonance imaging

- brain deposition

Magnetic resonance imaging has continued to evolve over the recent decades, in part, due to the evolution of gadolinium-based contrast agents and their use. These were initially thought to have a relatively low-risk profile. However, there is mounting evidence that trace amounts of gadolinium are retained within the body. To ascertain the current use of gadolinium in medical practice, we performed a survey of musculoskeletal radiologists, within the United Kingdom, Europe and India. The survey demonstrated varied practices amongst all radiologists with relatively indiscriminate use of gadolinium. In this review, we discuss the current evidence for and against the use of gadolinium in musculoskeletal magnetic resonance imaging.
\end{abstract}

\section{Background}

Magnetic resonance imaging (MRI) is a sensitive noninvasive modality with several advantages in comparison to other imaging techniques. ${ }^{1}$ It is the primary imaging modality for the detailed evaluation of a broad spectrum of musculoskeletal (MSK) disease processes. ${ }^{2}$ This is due to its highresolution providing unparalleled soft tissue contrast and allowing the visualization of both anatomical structures and pathological processes. ${ }^{3}$

The modality of choice for tumors and tumor-like conditions is often MRI, owing to its excellent soft tissue contrast, its sensitivity to bone marrow and soft tissue edema, and its multiplanar imaging. ${ }^{1,4}$ It is key for diagnosing, staging, preoperative work-up, and follow-up of patients with benign and malignant soft tissue neoplasms. ${ }^{3-5}$ Furthermore, it provides detailed tissue characterization and aids in the staging of bone lesions. $^{2}$ MRI is also useful in the evaluation of trauma, ${ }^{2,6}$ infection, and neuromuscular disease. ${ }^{3}$
The 1980s brought about new advances in MSK MRI with the development of gadolinium-based contrast agents (GBCAs). ${ }^{7}$ In its free form, unpaired gadolinium electrons are highly toxic. ${ }^{8}$ Thus, to reduce their toxicity and improve stability, they are bound to a ligand and administered in chelated forms. ${ }^{8,9}$

The use of GBCAs has grown substantially; they are used in approximately one-in-three of all MRI studies worldwide. ${ }^{9,10}$ GBCAs are used in an attempt to improve diagnostic confidence to influence patient care and management. ${ }^{11}$ In MSK MRI GBCAs are often used in the assessment of soft tissue sarcomas (STS) prior to histological diagnosis and in follow-up imaging to assess for local recurrence. ${ }^{12}$ They help radiologists plan soft tissue biopsies by identifying viable enhancing malignant tissue from cystic/necrotic tissue. ${ }^{11}$ Contrast can enable the detection of the early stages of soft tissue infection and differentiate phlegmon from normal surrounding tissues. ${ }^{13}$ It is also useful to gauge the extent of the infections and make abscess/collections more conspicuous. ${ }^{13}$ GBCAs can provide accurate representation of
DOI https://doi.org/ 10.1055/s-0041-1735500. ISSN 0971-3026.

\footnotetext{
(C) 2021. Indian Radiological Association. All rights reserved. This is an open access article published by Thieme under the terms of the Creative Commons Attribution-NonDerivative-NonCommercial-License, permitting copying and reproduction so long as the original work is given appropriate credit. Contents may not be used for commercial purposes, or adapted, remixed, transformed or built upon. (https://creativecommons.org/ licenses/by-nc-nd/4.0/) Thieme Medical and Scientific Publishers Pvt. Ltd., A-12, 2nd Floor, Sector 2, Noida-201301 UP, India
} 
Table 1 A simple anonymous yes/no web-based survey of eight questions was sent to members of British Society of Skeletal Radiologists (BSSR), European Society of Musculoskeletal Radiology (ESSR), and the Musculoskeletal Society of India (MSS) regarding the use of gadolinium use in MSK MRI

\begin{tabular}{|c|c|c|c|c|c|}
\hline \multicolumn{6}{|c|}{ Survey sent to BSSR/ESSR/MSS } \\
\hline No. & Questions & \multicolumn{4}{|l|}{ Choices } \\
\hline 1 & Do you use contrast in musculoskeletal MR imaging? & \multicolumn{2}{|l|}{ Yes } & \multicolumn{2}{|l|}{ No } \\
\hline 2 & $\begin{array}{l}\text { Relative proportion of post contrast } \\
\text { imaging in your practice? }\end{array}$ & $<25 \%$ & $25-50 \%$ & & $>50 \%$ \\
\hline 3 & Post contrast imaging in soft tissue lump? & \multicolumn{2}{|l|}{ Yes } & \multicolumn{2}{|l|}{ No } \\
\hline 4 & Post-contrast imaging in bone lesions? & \multicolumn{2}{|l|}{ Yes } & \multicolumn{2}{|l|}{ No } \\
\hline 5 & Post-contrast imaging in soft tissue infection? & \multicolumn{2}{|l|}{ Yes } & \multicolumn{2}{|l|}{ No } \\
\hline 6 & Post-contrast imaging in bone infection? & \multicolumn{2}{|l|}{ Yes } & \multicolumn{2}{|l|}{ No } \\
\hline 7 & Where do you work? & $\begin{array}{l}\text { Tertiary referral } \\
\text { center }\end{array}$ & $\begin{array}{l}\text { University } \\
\text { hospital }\end{array}$ & $\begin{array}{l}\text { District } \\
\text { hospital }\end{array}$ & Private clinic \\
\hline 8 & Do you check eGFR before administration of contrast? & \multicolumn{2}{|l|}{ Yes } & \multicolumn{2}{|l|}{ No } \\
\hline
\end{tabular}

Abbreviation: eGFR, estimated glomerular filtration rate.

the degree of osseous and nonosseous involvement in complicated extremity infections. ${ }^{14}$ Additionally, GBCAs can aid in the diagnosis of infections in septic arthritis, acutesubacute- and chronic osteomyelitis. ${ }^{13}$ The role of GBCAs in spinal disease will not be covered in this article.

\section{Mechanism of Action}

Gadolinium $\left(\mathrm{Gd}^{3+}\right)$, in its raw form is a paramagnetic ion composed of seven unpaired electrons ${ }^{1}$ resulting in a highly magnetic effect. Administration of $\mathrm{Gd}^{3+}$ falters the rotation frequency of water molecules, shortening both T1 and T2 relaxation times of tissues in which it accumulates, thus allowing differentiation through increasing signal intensity on $\mathrm{T} 1$ sequences and decreasing signal on T2 sequences. ${ }^{15}$ GBCAs are distributed within the blood and extravascularextracellular space. $^{16}$ They are biologically inert and generally eliminated by the kidneys. ${ }^{1}$

As previously mentioned, $\mathrm{Gd}^{3+}$ in its free form is highly toxic. Its structure makes it unstable in vivo and therefore it is bound to a ligand and administered in chelated forms. ${ }^{1}$ Pharmacologically GBCAs are classified according to the molecular structure of the chelating ligand to which they are bound. These are classified as linear or macrocyclic. ${ }^{17}$ The chelating ligand compounds are designed to minimize dissociation of gadolinium. It is because of this; GBCAs were expected to have high contrast efficiency and safety in addition to their rapid excretion, high stability, low osmolality, and low viscosity. ${ }^{17}$

\section{Current Practice}

In an attempt to assess current practices regarding the use of GBCAs, we anonymously surveyed members of the British Society of Skeletal Radiologists (BSSR), European Society of Musculoskeletal Radiology (ESSR), and the Musculoskeletal Society of India (MSS). Eight multiple-choice questions were asked ( - Table $\mathbf{1}$ ) via a simple web-based survey platform. A total of 100 BSSR members responded with 83 from ESSR/MSS. From the total responses, 95\% (172/182) stated GBCAs were used for MSK MRI; this was predominantly for less than $25 \%$ of cases, with a few stating more than $50 \%$ of imaging involves GBCAs. $87 \%$ responded with the use of GBCAs for soft tissue lumps. Reviewing detailed responses from those who answered "yes," demonstrated this ranged from $<5$ to $100 \%$ of cases for lumps. The use of GBCAs was more common amongst ESSR/MSS respondents with $97 \%$ using GBCAs for soft tissue lumps. Many respondents stated that peripheral hospitals perform contrast-enhanced MRI (CEMRI) for the assessment of lumps, as these patients are referred to a specialist hospital and administrating GBCAs is often the protocol. $88 \%$ of ESSR/MSS respondents said they use GBCAs for bone lesions compared with $47 \%$ of BSSR members. A total of $86 \%$ of those surveyed use GBCAs for soft tissue infections and $77 \%$ for bone infection. 5 to $8 \%$ of responders do not check renal function prior to gadolinium use. Selected results from the survey are shown in -Figs. 1 to 3.

\section{Current Evidence of GBCAs Deposition}

In addition to its key role in the development of nephrogenic systemic fibrosis (NSF), there is evidence of $\mathrm{Gd}^{3+}$ deposition in patients receiving GBCAs despite having an intact bloodbrain barrier and normal renal function. ${ }^{18}$ This was first reported in 2010 by Xia et al with the discovery of insoluble deposits of gadolinium in the biopsies of brain tumor patients, all of whom had at least one CEMRI scan with a linear chelating agent in their past. ${ }^{19}$ In 2013, Kanda et al reported an association between GBCAs administration and retention in deep brain nuclei. ${ }^{20}$ Studies found this to be connected with changes of the subcortical gray matter on MRI of the brain. ${ }^{21}$ Unenhanced T1-weighted images showed a positive correlation with previous exposure to nonionic 


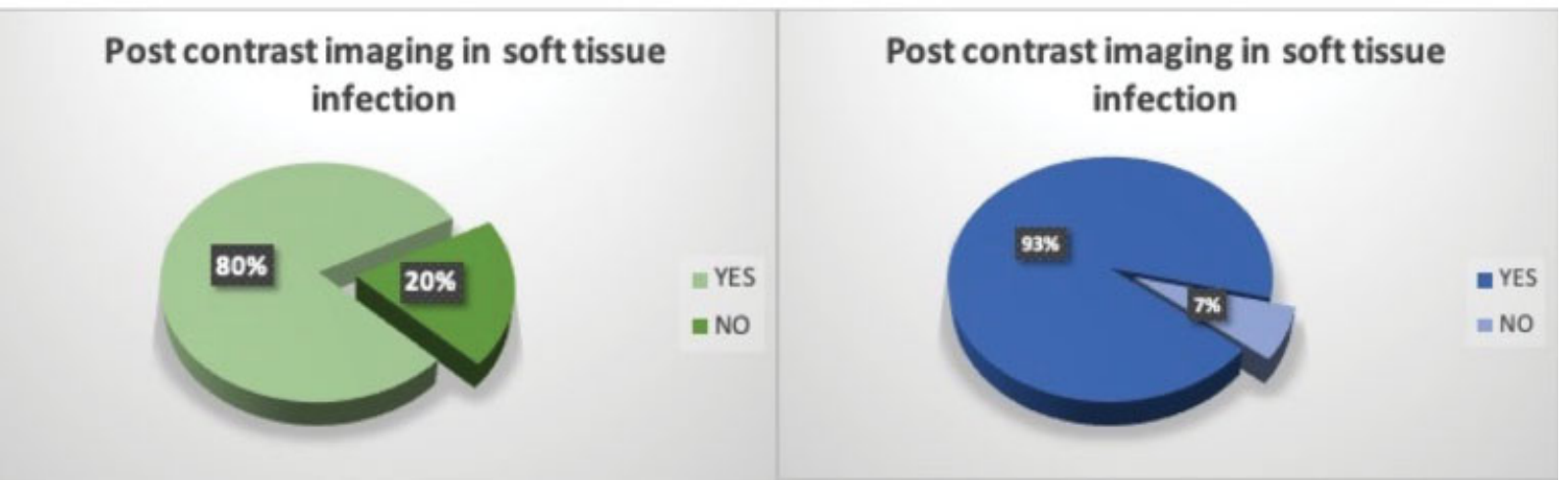

Fig. 1 Survey response from members of British Society of Skeletal Radiologists (BSSR), European Society of Musculoskeletal Radiology (ESSR), and the Musculoskeletal Society of India (MSS) demonstrating that the majority of respondents use gadolinium-enhanced MRI for the assessment of soft tissue infection.

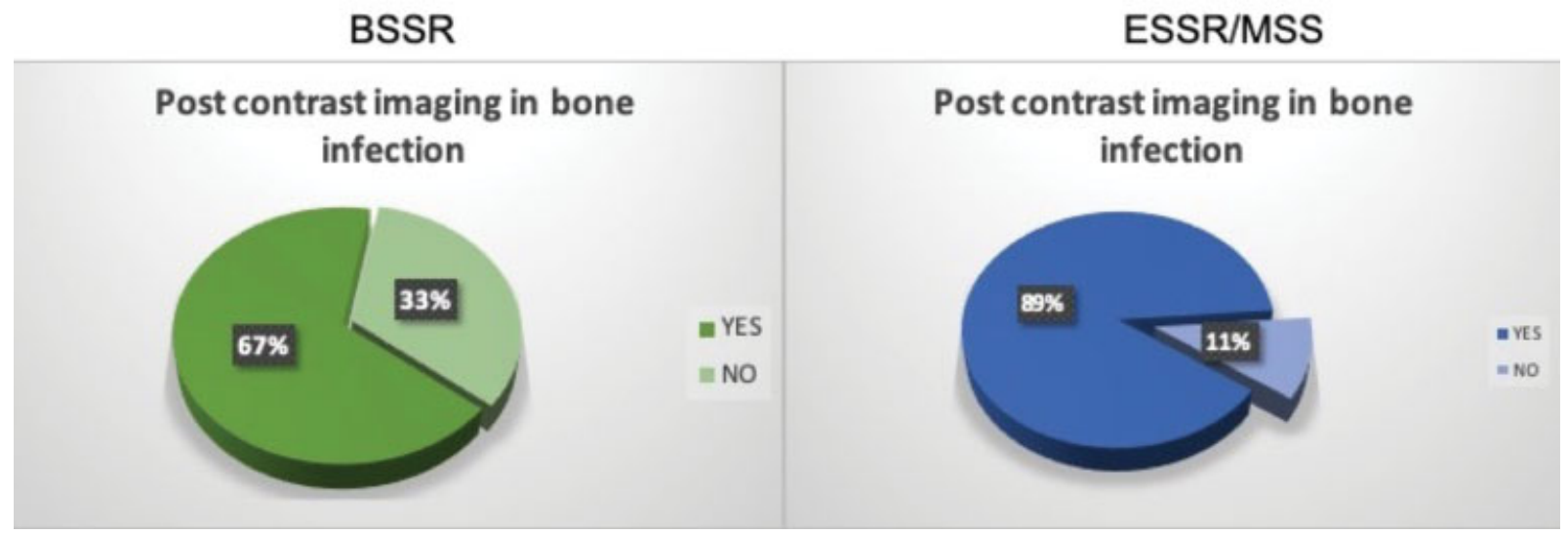

Fig. 2 Survey response from members of British Society of Skeletal Radiologists (BSSR), European Society of Musculoskeletal Radiology (ESSR), and the Musculoskeletal Society of India (MSS) demonstrating that the majority of respondents use gadolinium-enhanced MRI for the assessment of bone infection.

BSSR

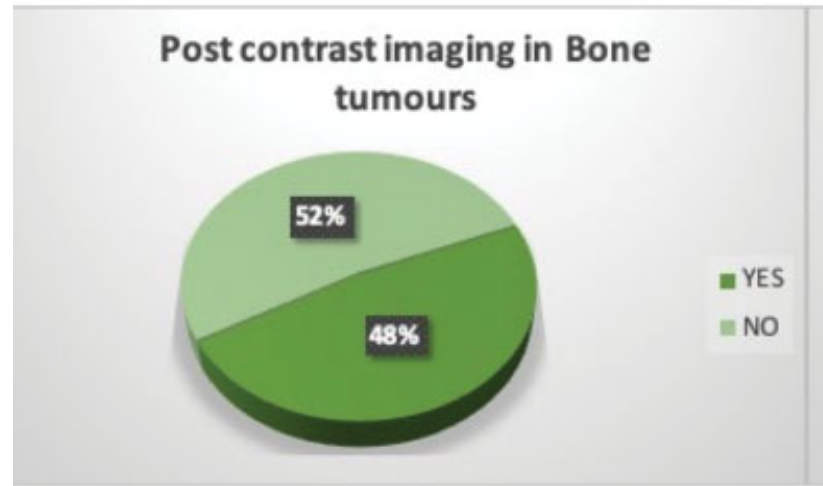

ESSR/MSS
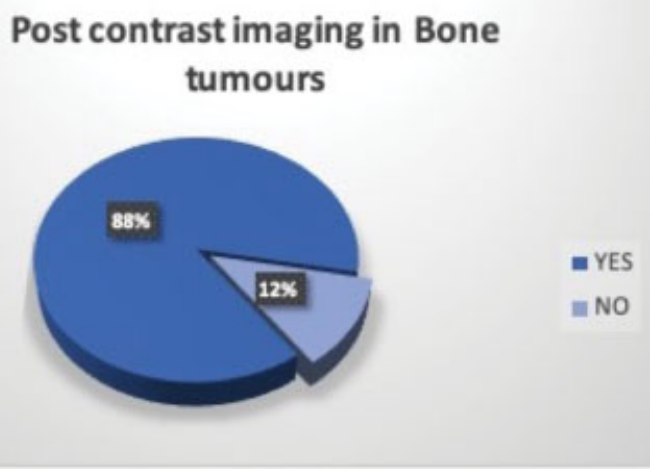

Fig. 3 Survey response from members of British Society of Skeletal Radiologists (BSSR), European Society of Musculoskeletal Radiology (ESSR), and the Musculoskeletal Society of India (MSS) regarding the use of gadolinium in bone tumors.

linear chelating type GBCAs, demonstrating areas of highintensity signals bilaterally in the globus pallidus (GP) and in the dentate nuclei (DN). ${ }^{21,22}$ Extracranial sites of gadolinium deposition have been reported in the liver, skin, and bones. ${ }^{23}$ Previously, it has been suggested in studies by Roccatagliata and colleagues, that multiple sclerosis was associated with hyperintense DN presence on MRI. ${ }^{24}$ Similarly, Kasahara et al proposed an association between hyperintense DN findings with a history of brain irradiation. ${ }^{25}$ However, Kanda et al found these changes were in fact associated with previous 
GBCAs administrations, rather than any relation to history of multiple sclerosis or brain irradiation. ${ }^{20}$ This was further supported by Ranga et al as hyperintense DN findings in irradiated patients were found to be likely related to gadolinium deposition. ${ }^{18}$

In addition, a positive dose-response correlation between the number of previous GBCAs administrations, and high signal intensity in the DN and GP was established by Kanda et al. ${ }^{20}$ Similar findings in pediatric patients were illustrated in case reports by Miller et al and Roberts and Holden, in which cumulative doses of administered GBCAs demonstrate significant changes in signal intensity. ${ }^{26,27}$

Brain specimens of patients with a history of receiving linear GBCAs were evaluated along with a control group. These studies revealed that there were increased gadolinium deposits in the GP and DN compared with other brain regions. ${ }^{28-30}$ Several studies by Radbruch et al and others support the hypothesis that gadolinium accumulation in the deep brain nuclei is associated with linear GBCAs and not macrocyclic GBCAs. ${ }^{29-33}$

\section{Risks and Side Effects of Gadolinium Deposition}

Apart from the rare incidence of NSF, the potential impact of long-term $\mathrm{Gd}^{3+}$ retention remains unknown. The risk of developing adverse effects following gadolinium deposition in the brain is significantly increased in patients who are subjected to multiple scans throughout their lifetime, using GBCAs. ${ }^{21}$ Those with chronic conditions who undergo serial surveillance scans or patients who have interval follow-up scans are at increased risks of gadolinium brain deposition. ${ }^{34}$ In addition, young children due to their age and expected long lifespan, bear considerable risk and should, therefore, be given the appropriate consideration and risk assessment to minimize exposure and deposition. ${ }^{34}$

A large prospective cohort study with a control group was designed by Parillo et al to evaluate the occurrence of symptoms within 24 hours after GBCA administration. These symptoms have been grouped as gadolinium deposition disease (GDD). Findings showed an increased incidence of new symptoms within the first 24 hours subsequent to GBCA exposure, in comparison to after unenhanced MRI. ${ }^{35}$ Patients reported symptoms of fatigue, dizziness, mental confusion, and diarrhea. ${ }^{35}$ In a separate study, performed by Burke et al which consisted of anonymous patient surveys, 66\% of respondents self-reported immediate adverse manifestations experienced following GBCA administration, 32\% within 6 weeks, and $2 \%$ complained of symptoms within a 6-month period. ${ }^{36}$ More than $77 \%$ reported side effects such as headaches, visual changes, auditory changes, and bone/joint pains. ${ }^{36}$ Skin changes, such as thickening and discoloration, were reported by $>50 \%$ of those surveyed, while respiratory (difficulty in breathing) and digestive (nausea, vomiting, diarrhea) changes were felt by $>40 \%{ }^{36}$ All respondents related their symptoms to their previous GBCA exposure. Although this survey suggests a temporal relationship between gadolinium and the reported symptoms, the invalidity of self-report surveys indicated that further research is advised.

With the DN being the most noted site of gadolinium deposition, adverse effects relating to its functions of planning, initiation, and control of voluntary movements are expected. ${ }^{21}$ However, none have been reported in relation to any GBCA exposure.

Well-controlled studies to investigate the adverse biological and/or neurological side effects of GBCAs administration are essential both to conclude the short- and long-term effects of gadolinium deposition in the brain. In addition, data linking these adverse effects to gadolinium deposition in the brain must also be established. Despite being unproven scientifically, there has been an increase of GDD-related litigation and personal injury advertising in the United States of America targeting potential GDD patients.

\section{Regulatory Changes Related to GBCA}

With the emergence of new evidence regarding GBCAs retention in the brain, guidelines have been amended after investigations in 2016 by the Pharmacovigilance Risk Assessment Committee (European Medicines Agency) and submitted recommendations to the Committee of Medicinal Products for Human Use in 2017. ${ }^{37}$ As a result, the Royal College of Radiologists has updated its guidance on the use of $\mathrm{Gd}^{3+}{ }^{(8)}$. The new guidelines highlight the suspension, withdrawal, or alterations in the use of some linear chelate GBCAs. ${ }^{7}$ The changes highlight the need to reconsider the routine use of gadolinium unless the diagnostic need outweighs possible unknown future complications.

\section{Gadolinium in Food}

Research in Germany has found traces of gadolinium in beverages such as Coca-Cola. ${ }^{38}$ This has been attributed to gadolinium in the urine excreted by patients post-CEMRI and entering municipal wastewater treatment systems. Gadolinium is not removed or purified by the treatment systems and as a result enters the public water supply and subsequently the environment. Schmidt et al reported six major German cities had gadolinium polluted water systems and present in food and beverages from McDonald's and Burger King. ${ }^{38}$ In addition, Thomsen noted that the gadolinium concentration levels are rising slowly and remain persistent in water thus causing a growing concern. ${ }^{39}$ Currently, no clinical adverse effects have been reported although the long-term implications remain unknown.

\section{Current Evidence Supporting the Use of Gadolinium}

Currently, the use of gadolinium in MSK MR can be classified into three main groups: tumors, infections, and joint pathology. 


\section{Soft Tissue Sarcomas}

In the evaluation of sarcomas and sarcoma like-lesions, gadolinium is thought to improve diagnostic accuracy and provide additional data in staging, biopsy planning, tissue characterization, evaluation of response to chemotherapy, and detection of recurrence. ${ }^{40}$ Gadolinium has been reported to increase the sensitivity of recurrent STS by $74 \%$ compared with unenhanced MRI. ${ }^{41}$ GBCAs allow the assessment of intra- or extra-compartmental extent and involvement of adjacent bone, joint, muscle, or neurovascular involvement. In addition, biopsy planning is made easier by improved tissue enhancement highlighting necrotic and/or cystic areas to be avoided. ${ }^{41}$ It can be difficult to distinguish between true cystic lesions and cystic-like solid lesions on noncontrast MRI (e.g., myxoid lesions); gadolinium allows this distinction to be readily made due to the lack of enhancement of true cystic lesions. ${ }^{40,42}$ One relies on tumor enhancement to make tumors more conspicuous when there is significant peritumoral edema. ${ }^{11,42}$ Gadolinium can also be of value in the evaluation of hemorrhagic lesions. It will uncover enhancing tumors masked by the surrounding hemorrhage. ${ }^{40,43}$ Dynamic contrast enhanced (DCE) MRI is a technique that allows the evaluation of the temporal pattern of enhancement in tumors, monitoring response to neoadjuvant chemotherapy, and assessing for tumor recurrence. Other uses include distinguishing adjacent inflammatory processes and bone tumor perfusion. ${ }^{44}$ Postoperative gadolinium is useful in distinguishing underlying collections from surrounding inflammatory change and recurrence.

\section{Bone Tumors}

With regard to bone tumors MRI is primarily performed for local staging and extent rather than diagnosis and therefore there is no requirement of contrast. Radiological diagnosis is predominantly based on radiography. However, GBCAs may provide information on the assessment of intramedullary extension, the extension to adjacent structures and can be useful in post-surgical follow-up imaging. ${ }^{45,46}$ For selective bone tumors, such as osteosarcoma, gadolinium offers the potential for determining the efficacy of chemotherapy, by evaluating tumor necrosis prior and subsequent to chemotherapy. Sarcomas close to joints, gadolinium may aid in determining whether tumor resection should be intra- or extra-articular. ${ }^{50}$ One may need to give contrast when faced with equivocal imaging findings for suspected osteoid osteomas (OO), as dynamic MRI increases nidus conspicuity. ${ }^{47}$ It should be noted that in our center of 793 cases of 00 over a 12-year period, we have never needed to use dynamic imaging for cases of 00 . The results of our survey demonstrate that a large proportion of radiologists are using GBCAs for bone lesions, and this is disproportionately higher in Europe/India.

\section{Arthritis}

Several studies have shown that GBCA-enhanced MRI is beneficial in discriminating active from dormant arthritis. ${ }^{48-51}$
For tenosynovitis, sensitivity and specificity are decreased without gadolinium contrast administration. Gadolinium contrast administration increases sensitivity when evaluating synovitis and tenosynovitis in early arthritis. ${ }^{52} \mathrm{~A}$ study by Reiser and coworkers examining both knee and wrist joints showed the use of gadolinium contrast markedly increased the enhancement between pannus and effusion, improving detection. ${ }^{48}$ In addition, CEMRI improved the evaluation of the pannus extension in the joint cavity, and into the suprapatellar recess. Furthermore, GBCA was effective in tracking the therapeutic effectiveness of treatment and found to be valuable in the selection process of patients suitable for synovectomy. ${ }^{48,49}$ König and colleagues were able to differentiate between fibrous, slightly hypervascular, and hypervascular pannus using GBCAs. ${ }^{53}$

\section{Infections}

Gadolinium's role was suggested as an aid to clarify the extent of active infections, distinguishing infectious from noninfectious inflammatory lesions, and in highlighting soft tissue abscesses. ${ }^{54}$ Characterization of focal collections and differentiation of abscesses from surrounding cellulitis/myositis were both improved by gadolinium. ${ }^{54}$ In septic arthritis, CEMRI was found to be useful in the evaluation of synovial hypertrophy. ${ }^{40}$ In addition, synovitis was more easily differentiated from simple joint effusion using GBCAs. ${ }^{11}$ Hopkins et al showed that the major role of CEMRI lies in diagnosing osteomyelitis and distinguishing it from neuropathic disease. ${ }^{54} \mathrm{Gd} 3+$ can be especially useful when imaging the diabetic foot and to help differentiate osteomyelitis from Charcot's arthropathy. Differentiating between the two requires careful evaluation of the patient, including medical history, physical examination, selected laboratory findings, and imaging studies. The use of contrast demonstrates areas of nonenhancement amongst enhancing inflammatory tissue allow necrotic regions in bone or abscesses to become more conspicuous and suggestive of osteomyelitis. However, one must proceed with caution if administrating gadolinium due to potential complications with contrast nephropathies in poorly controlled diabetic patients.

\section{Joint Pathologies}

The evaluation of internal joint pathology with the use of gadolinium has been well established. Intra-articular $\mathrm{Gd}^{3+}$ aids in the assessment of labrum or cartilage. ${ }^{40} \mathrm{MR}$ arthrography has advantages over conventional MR imaging owing to distention of the joint capsule, outlining the intra-articular structures, and hence delineating the abnormalities. ${ }^{55}$ This can be achieved by direct injection into the joint or indirect via intravenous gadolinium administration.

\section{Current Evidence against the Use of Gadolinium}

\section{Tumors}

Numerous studies have shown that gadolinium has done little to improve the diagnostic specificity of MRI. May et al 


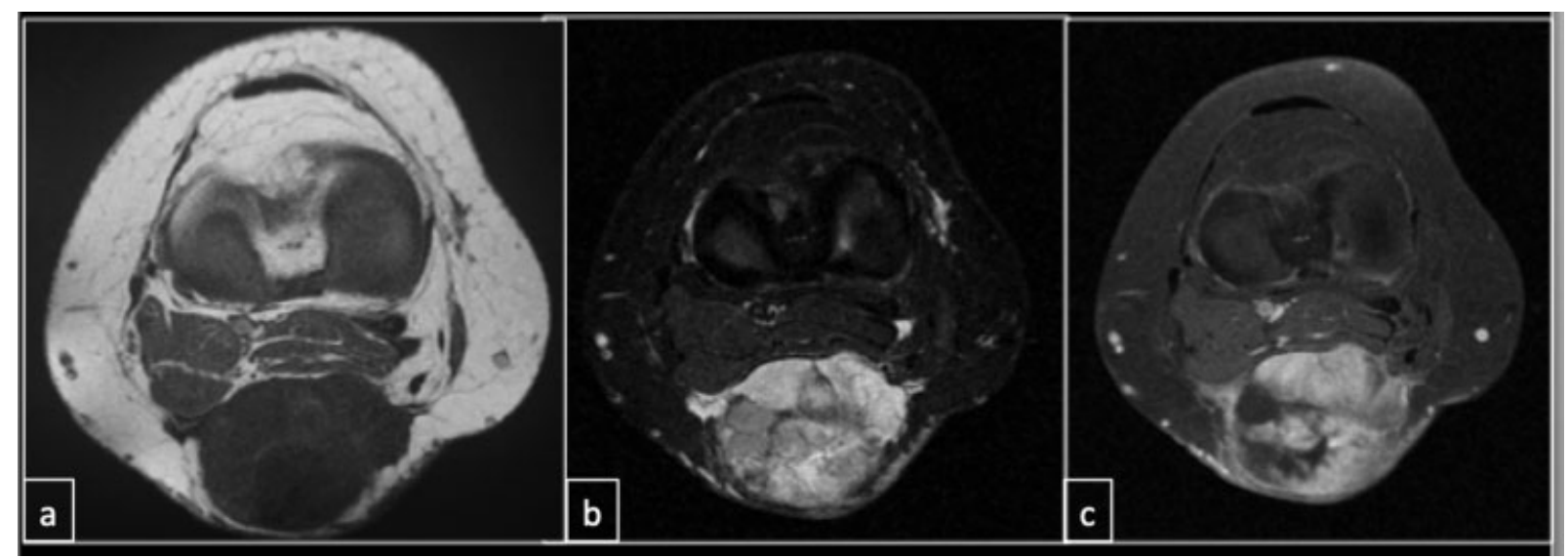

Fig. 4 Axial MRI (a) T1, (b) STIR, and (c) T1FS post-contrast of a 63-year-old female with histologically proven undifferentiated pleomorphic sarcoma. There is an aggressive appearing soft tissue lesion which will require biopsy. The post-contrast image (c) confirms the mass is solid and demonstrates nonenhancing necrotic areas within the tumor. Ultrasound can clearly demonstrate both solid and necrotic components (image not shown) thus allowing a biopsy to be obtained from the most appropriate region of the lesion. The addition of the gadolinium, in this case, did not provide additional useful diagnostic information in either making the diagnosis or deciding if the lesion required a biopsy. MRI, magnetic resonance imaging.

reported CEMRI did not provide additional information in $89 \%$ of cases and only led to changes in the management in $\leq 10 \%$ of patients ( - Fig. 4 ).$^{11}$ Gadolinium-enhanced imaging did not lead to a reliable distinction between lesions, surrounding edema and fibrovascular tissue present in organizing hematomas which may show enhancement similar to tumor nodules. ${ }^{11,41}$ Additionally, cystic regions have particular signal characteristics on noncontrast images which should be identifiable, particularly if a fluid-fluid level is present (-Fig. 5). The associated costs of GBCAs and increased length of examination associated with the acquisition of specific post-contrast sequences must also be considered. ${ }^{40}$ Furthermore, patients may not tolerate the increased length of scanning time, risking image degradation from movement artifact. It can be argued that the routine use of GBCAs for the evaluation of soft tissue tumors has negligible benefits. Its effectiveness in the evaluation and staging of MSK neoplasms is controversial. GBCAs should only be administered if it will change management. Indeterminate or aggressive appearing soft tissue lesions will often undergo an image-guided biopsy. If MRI demonstrates cystic/necrotic areas, ultrasound will allow the identification of solid areas to target, therefore, negating the need for GBCA use (-Fig. 6). GBCAs rarely provide additional information during the assessment of primary bone lesions ( - Fig. 7).

\section{Infections}

Gadolinium does not allow radiologist to reliably distinguish infectious from noninfectious inflammatory conditions. Although evidence has shown gadolinium-enhanced MRI to be a highly sensitive (89-100\%) technique in diagnosing MSK infections, the use of GBCAs varies in specificity from 46 to
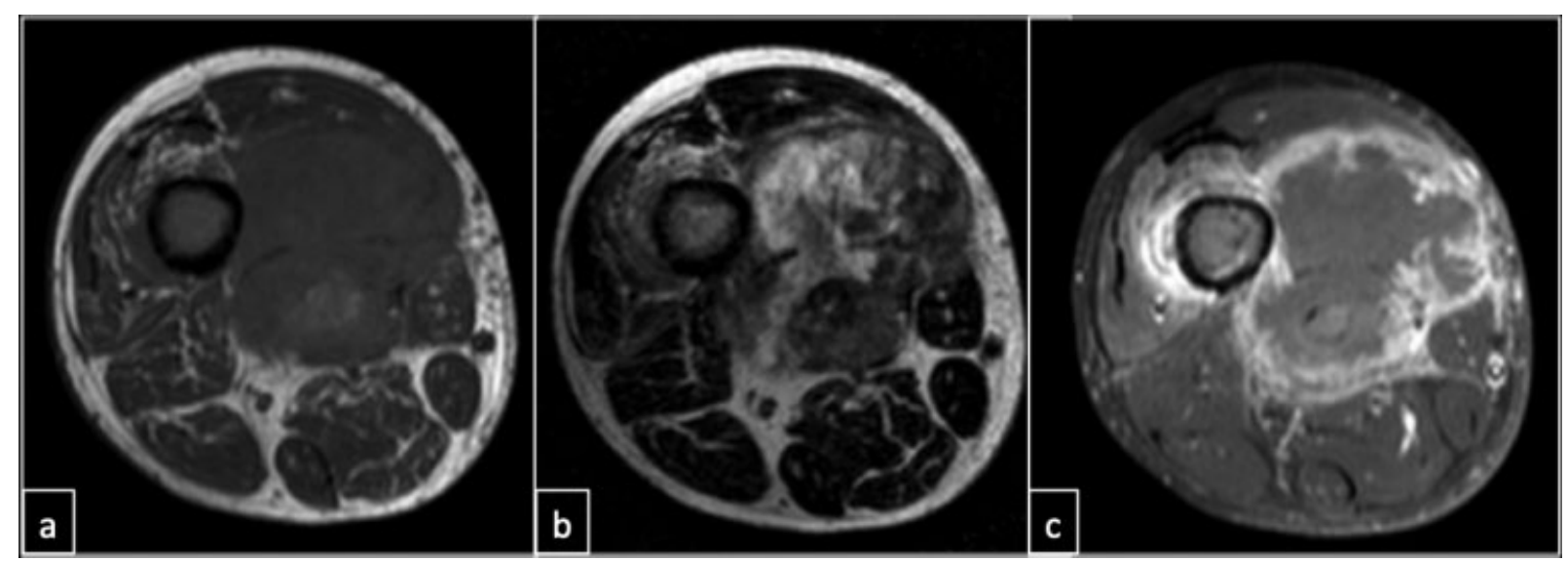

Fig. 5 Axial MRI (a) T1, (b) T2, and (c) T1FS post-contrast of a 74-year-old male with histologically proven undifferentiated pleomorphic sarcoma. This is a predominantly necrotic lesion with areas of hemorrhage shown as intralesional areas of high T1 signal. The low T1/high T2 areas are necrotic/cystic areas and the enhancing wall confirms the solid component as seen in (c). However, gadolinium use did not provide information on the type of aggressive mass and solid areas for target for biopsy seen on ultrasound (image not shown). MRI, magnetic resonance imaging. 


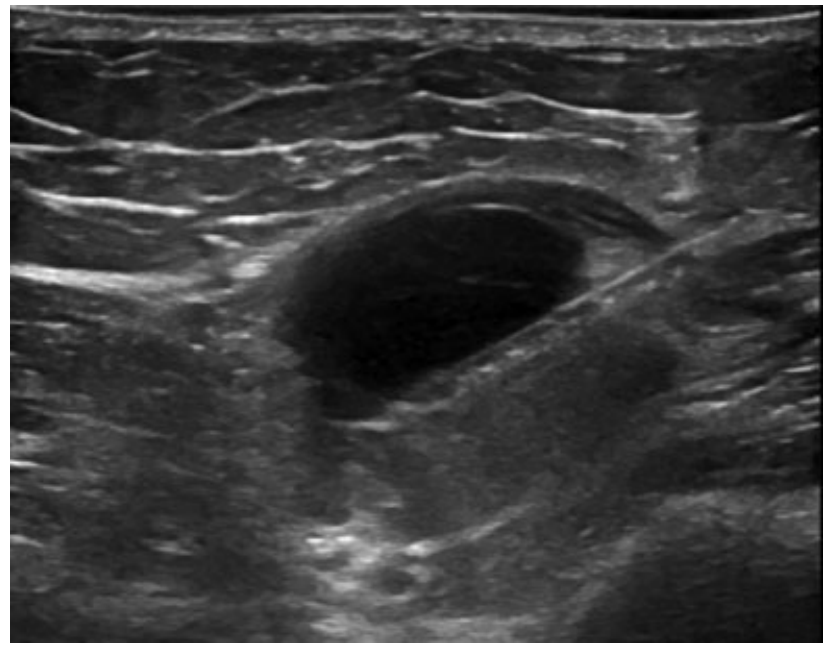

Fig. 6 Transverse greyscale ultrasound image of a soft tissue lesion in another patient with histologically proven sarcoma. The superior aspect is anechoic in keeping with necrosis with the solid inferior part of the lesion representing viable tissue. A biopsy needle is targeted into this area. Administration of gadolinium would not have been of clinical value.

$88 \%$ and as a result often does not lead to alterations in patient care. $^{54}$

\section{Arthritis and Joint Pathologies}

GBCA can be useful in demonstrating the enhancement of the synovium but is unable to differentiate between similar inflammatory lesions. ${ }^{56}$ Rheumatoid and septic arthri- tis show similar enhancements. ${ }^{57}$ Doppler ultrasound should be considered in the first instance for the assessment of tenosynovitis/synovitis. Diffusion-weighted imaging has shown promising results in detecting synovitis and may be a novel noninvasive approach to contrast-free imaging of synovitis.$^{58}$ The resolution of 3T MRI is such that there is mounting evidence supporting the use of unenhanced 3T MRI to evaluate the hip labrum. ${ }^{59,60}$ Evidence is not as clear for the glenoid labrum. ${ }^{61-64}$ The lack of $3 \mathrm{~T}$ magnets and expertise in image interpretation means traditional arthrograms are still performed. In the future, arthrograms, particularly of the hip and shoulder, may become obsolete as 3T imaging becomes the standard. 3T MRI allows higher resolution and implements a small field of view strategies to improve spatial resolution, negating the need for contrast.

\section{Conclusion}

It is important to recognize the role of gadolinium in specific clinical settings such as infection and post-surgical follow-up of soft tissue tumors (-Table 2). However, the use of GBCAs in MSK imaging is not without controversy and as innocuous as previously thought. Despite the unknown clinical implications, the mounting evidence of deposition in the body, contamination of water supplies as well as the food chain, and potential medicolegal implications, one should give due consideration before proceeding with its use. In conclusion, the use of GBCAs should be assessed on a case-by-case basis depending on the clinical scenario and merit rather than routine protocol.
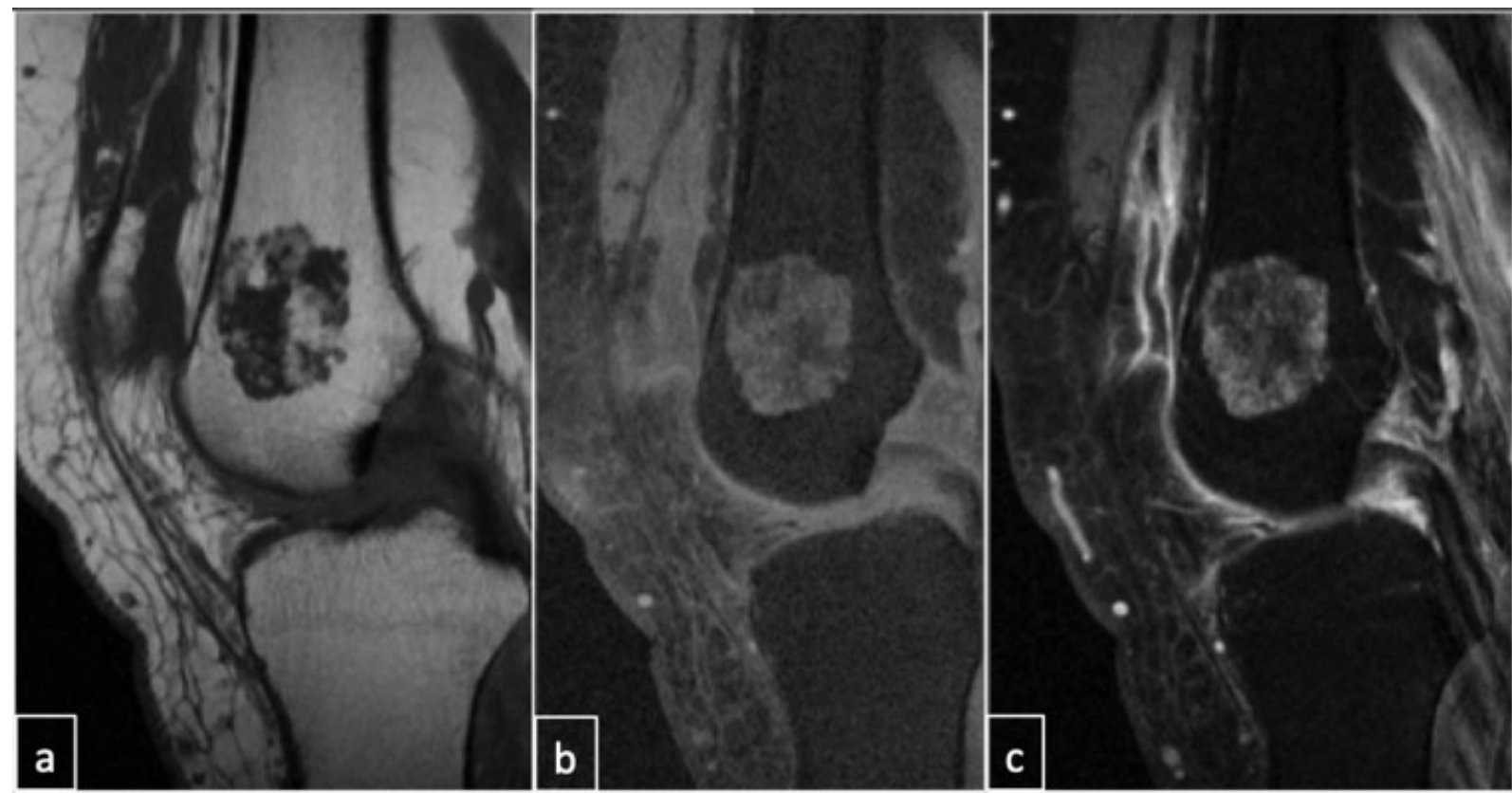

Fig. 7 Sagittal MRI (a) T1, (b) T1FS pre-, and (c) T1FS post-contrast of a 61-year-old female with classical appearances of an enchondroma. The intramedullary lesions has the classic intramedullary location and cartilaginous matrix. The addition of gadolinium has not altered the radiological diagnosis. The appearances correlated with radiographs (not shown) confirm the diagnosis. The enhanced images also demonstrate no aggressive features to suggest sarcomatous change and again, this would not be different on post-contrast images. MRI, magnetic resonance imaging. 
Table 2 Summary of proposed recommendation for the use of gadolinium in MSK MRI

\begin{tabular}{|l|l|}
\hline $\begin{array}{l}\text { Low-level indications for the use of } \\
\text { gadolinium: }\end{array}$ & $\begin{array}{l}\text { - Bone tumors (exception of dynamic imaging for osteoid osteoma if } \\
\text { required). } \\
\text { - Solid soft tissue tumors (except for necrotic or myxoid cases where } \\
\text { ultrasound is not available). } \\
\text { - Trauma or internal derangements (joints). }\end{array}$ \\
\hline $\begin{array}{l}\text { Intermediate level indications for the use of } \\
\text { gadolinium: }\end{array}$ & $\begin{array}{l}\text { - Arthritis (need to differentiate synovitis/pannus from effusions). } \\
\text { - Diabetic foot. }\end{array}$ \\
\hline $\begin{array}{l}\text { High-level indications for the use of } \\
\text { gadolinium: }\end{array}$ & $\begin{array}{l}\text { - Necrotic soft tissue tumors (to aid biopsy planning if ultrasound not } \\
\text { available). }\end{array}$ \\
\hline
\end{tabular}

Abbreviation: MRI, magnetic resonance imaging; MSK, musculoskeletal.

Note: The use of gadolinium has been grouped into low, intermediate, and high-level indications. A low level represents cases where gadolinium should not be used, as no added clinical benefit is seen. Intermediate and high levels represent cases which may provide additional benefit with the use of gadolinium; however, this should be considered on a case-by-case basis.

\section{Funding \\ None.}

\section{Conflict of Interest \\ None declared.}

\section{References}

1 Lohrke J, Frenzel T, Endrikat J, et al. 25 years of contrast-enhanced MRI: developments, current challenges and future perspectives. Adv Ther 2016;33(01):1-28

2 Ma LD. Magnetic resonance imaging of musculoskeletal tumors: skeletal and soft tissue masses. Curr Probl Diagn Radiol 1999;28(02):29-62

3 Dean Deyle G. The role of MRI in musculoskeletal practice: a clinical perspective. J Manual Manip Ther 2011;19(03):152-161

4 Khedr S, Hassaan M, Abdelrazek N, Sakr A. Diagnostic impact of echo planar diffusion-weighted magnetic resonance imaging (DWI) in musculoskeletal neoplastic masses using apparent diffusion coefficient (ADC) mapping as a quantitative assessment tool. Egypt J Radiol Nucl Med 2012;43(02):219-226

5 Golfieri R, Baddeley H, Pringle JS, Leung AW, Greco A, Souhami R. MRI in primary bone tumors: therapeutic implications. Eur J Radiol 1991;12(03):201-207

6 Crues J, Bydder G. Frontiers in musculoskeletal imaging. J Magn Reson Imaging 2007;25(02):232-233

7 The Royal College of Radiologists. Guidance on gadoliniumbased contrast agent administration to adult patients. London Ref No. BFCR (19)4. Accessed August 27, 2021 at: https://www. rcr.ac.uk/publication/guidance-gadolinium-based-contrast-agentadministration-adult-patients

8 Lee DH. Mechanisms of contrast enhancement in magnetic resonance imaging. Can Assoc Radiol J 1991;42(01):6-12

9 Kanda T, Oba H, Toyoda K, Kitajima K, Furui S. Brain gadolinium deposition after administration of gadolinium-based contrast agents. Jpn J Radiol 2016;34(01):3-9

10 Runge V, Stewart R, Clanton J, Lukehart C, Partain C, James A. Work in progress: potential oral and intravenous paramagnetic NMR contrast agents. Magn Reson Imaging 1984;2(01):69

11 May DA, Good RB, Smith DK, Parsons TW. MR imaging of musculoskeletal tumors and tumor mimickers with intravenous gadolinium: experience with 242 patients. Skeletal Radiol 1997;26(01):2-15

12 Lo HH, Kalisher L, Faix JD. Epithelioid sarcoma:radiologic and pathologic manifestations. AJR Am J Roentgenol 1977;128(06): 1017-1020

13 Soldatos T, Durand DJ, Subhawong TK, Carrino JA, Chhabra A. Magnetic resonance imaging of musculoskeletal infections: systematic diagnostic assessment and key points. Acad Radiol 2012; 19(11):1434-1443
14 Towers JD. The use of intravenous contrast in MRI of extremity infection. Semin Ultrasound CT MR 1997;18(04):269-275

15 Caravan P, Farrar CT, Frullano L, Uppal R. Influence of molecular parameters and increasing magnetic field strength on relaxivity of gadolinium- and manganese-based T1 contrast agents. Contrast Media Mol Imaging 2009;4(02):89-100

16 Lusic H, Grinstaff MW. X-ray-computed tomography contrast agents. Chem Rev 2013;113(03):1641-1666

17 Runge VM, Ai T, Hao D, Hu X. The developmental history of the gadolinium chelates as intravenous contrast media for magnetic resonance. Invest Radiol 2011;46(12):807-816

18 Ranga A, Agarwal Y, Garg KJ. Gadolinium based contrast agents in current practice: risks of accumulation and toxicity in patients with normal renal function. Indian J Radiol Imaging 2017;27(02): 141-147

19 Xia D, Davis RL, Crawford JA, Abraham JL. Gadolinium released from MR contrast agents is deposited in brain tumors: in situ demonstration using scanning electron microscopy with energy dispersive X-ray spectroscopy. Acta Radiol 2010;51(10):1126-1136

20 Kanda T, Ishii K, Kawaguchi H, Kitajima K, Takenaka D. High signal intensity in the dentate nucleus and globus pallidus on unenhanced T1-weighted MR images: relationship with increasing cumulative dose of a gadolinium-based contrast material. Radiology 2014;270(03):834-841

21 Gulani V, Calamante F, Shellock FG, Kanal E, Reeder SBInternational Society for Magnetic Resonance in Medicine. Gadolinium deposition in the brain: summary of evidence and recommendations. Lancet Neurol 2017;16(07):564-570

22 Costa A, Ronchi A, Pigatto PD, Guzzi G. Brain gadolinium deposition, hyperintense MRI signals, and resonance contrast agents. Magn Reson Imaging 2018;52:137-138

23 Guo BJ, Yang ZL, Zhang LJ. Gadolinium deposition in brain: current scientific evidence and future perspectives. Front Mol Neurosci 2018; $11: 335$

24 Roccatagliata L, Vuolo L, Bonzano L, Pichiecchio A, Mancardi GL. Multiple sclerosis: hyperintense dentate nucleus on unenhanced T1-weighted MR images is associated with the secondary progressive subtype. Radiology 2009;251(02):503-510

25 Kasahara S, Miki Y, Kanagaki M, et al. Hyperintense dentate nucleus on unenhanced T1-weighted MR images is associated with a history of brain irradiation. Radiology 2011;258(01):222-228

26 Miller JH, Hu HH, Pokorney A, Cornejo P, Towbin R. MRI brain signal intensity changes of a child during the course of 35 gadolinium contrast examinations. Pediatrics 2015;136(06): e1637-e1640

27 Roberts DR, Holden KR. Progressive increase of T1 signal intensity in the dentate nucleus and globus pallidus on unenhanced T1weighted MR images in the pediatric brain exposed to multiple doses of gadolinium contrast. Brain Dev 2016;38(03):331-336 
28 McDonald RJ, McDonald JS, Kallmes DF, et al. Intracranial gadolinium deposition after contrast-enhanced MR imaging. Radiology 2015;275(03):772-782

29 Kanda T, Fukusato T, Matsuda M, et al. Gadolinium-based contrast agent accumulates in the brain even in subjects without severe renal dysfunction: evaluation of autopsy brain specimens with inductively coupled plasma mass spectroscopy. Radiology 2015; 276(01):228-232

30 Sanyal S, Marckmann P, Scherer S, Abraham JL. Multiorgan gadolinium (Gd) deposition and fibrosis in a patient with nephrogenic systemic fibrosis-an autopsy-based review. Nephrol Dial Transplant 2011;26(11):3616-3626

31 Radbruch A, Weberling LD, Kieslich PJ, et al. Gadolinium retention in the dentate nucleus and globus pallidus is dependent on the class of contrast agent. Radiology 2015;275(03):783-791

32 Cao Y, Huang DQ Shih G, Prince MR. Signal change in the dentate nucleus on T1-weighted MR images after multiple administrations of gadopentetate dimeglumine versus gadobutrol. AJR Am J Roentgenol 2016;206(02):414-419

33 Radbruch A, Weberling LD, Kieslich PJ, et al. High-signal intensity in the dentate nucleus and globus pallidus on unenhanced T1weighted images: evaluation of the macrocyclic gadoliniumbased contrast agent gadobutrol. Invest Radiol 2015;50(12): 805-810

34 Orenstein B. Gadolinium on the brain: curiosity or cause for concern? Radiol Today 2016;17(07):20

35 Parillo M, Sapienza M, Arpaia F, et al. A structured survey on adverse events occurring within 24 hours after intravenous exposure to gadodiamide or gadoterate meglumine: a controlled prospective comparison study. Invest Radiol 2019;54(04):191-197

36 Burke LM, Ramalho M, AlObaidy M, Chang E, Jay M, Semelka RC. Selfreported gadolinium toxicity: a survey of patients with chronic symptoms. Magn Reson Imaging 2016;34(08):1078-1080

37 PRAC. gadolinium deposition confirmed with linear agents. Reactions Weekly. 2017;1661(01):6

38 Schmidt K, Bau M, Merschel G, Tepe N. Anthropogenic gadolinium in tap water and in tap water-based beverages from fast-food franchises in six major cities in Germany. Sci Total Environ 2019; 687:1401-1408

39 Thomsen HS. Are the increasing amounts of gadolinium in surface and tap water dangerous? Acta Radiol 2017;58(03):259-263

40 Cheng SG. Musculoskeletal MRI: contrast and non-contrast applications. Appl Radiol 2002;31(06):81-86

41 Amini B, Murphy WA Jr, Haygood TM, et al. Gadolinium-based contrast agents improve detection of recurrent soft-tissue sarcoma at MRI. Radiol Imaging Cancer 2020;2(02):e190046

42 Drapé JL. Advances in magnetic resonance imaging of musculoskeletal tumours. Orthop Traumatol Surg Res 2013;99(Suppl 1): S115-S123

43 Kransdorf MJ, Murphey MD. The use of gadolinium in the MR evaluation of soft tissue tumors. Semin Ultrasound CT MR 1997; 18(04):251-268

44 Verstraete KL, Van der Woude HJ, Hogendoorn PC, De-Deene Y, Kunnen M, Bloem JL. Dynamic contrast-enhanced MR imaging of musculoskeletal tumors: basic principles and clinical applications. J Magn Reson Imaging 1996;6(02):311-321

45 Nascimento D, Suchard G, Hatem M, de Abreu A. The role of magnetic resonance imaging in the evaluation of bone tumours and tumour-like lesions. Insights Imaging 2014;5(04):419-440

46 Miwa S, Otsuka T. Practical use of imaging technique for management of bone and soft tissue tumors. J Orthop Sci 2017;22(03): 391-400

47 Sundaram M. The use of gadolinium in the MR imaging of bone tumors. Semin Ultrasound CT MR 1997;18(04):307-311
48 Reiser MF, Bongartz GP, Erlemann R, et al. Gadolinium-DTPA in rheumatoid arthritis and related diseases: first results with dynamic magnetic resonance imaging. Skeletal Radiol 1989;18 (08):591-597

49 Cimmino MA, Innocenti S, Livrone F, Magnaguagno F, Silvestri E, Garlaschi G. Dynamic gadolinium-enhanced magnetic resonance imaging of the wrist in patients with rheumatoid arthritis can discriminate active from inactive disease. Arthritis Rheum 2003; 48(05):1207-1213

50 Aoki T, Yamashita Y, Saito K, Tanaka Y, Korogi Y. Diagnosis of early-stage rheumatoid arthritis: usefulness of unenhanced and gadolinium-enhanced MR images at 3 T. Clin Imaging 2013;37 (02):348-353

51 Miese F, Buchbender C, Scherer A, et al. Molecular imaging of cartilage damage of finger joints in early rheumatoid arthritis with delayed gadolinium-enhanced magnetic resonance imaging. Arthritis Rheum 2012;64(02):394-399

52 Stomp W, Krabben A, van der Heijde D, et al. Aiming for a simpler early arthritis MRI protocol: can Gd contrast administration be eliminated? Eur Radiol 2015;25(05):1520-1527

53 König H, Sieper J, Wolf KJ. Rheumatoid arthritis: evaluation of hypervascular and fibrous pannus with dynamic MR imaging enhanced with Gd-DTPA. Radiology 1990;176(02):473-477

54 Hopkins KL, Li KC, Bergman G. Gadolinium-DTPA-enhanced magnetic resonance imaging of musculoskeletal infectious processes. Skeletal Radiol 1995;24(05):325-330

55 Steinbach LS, Palmer WE, Schweitzer ME. Special focus session. MR arthrography. Radiographics 2002;22(05):1223-1246

56 Zampa V, Bargellini I, Ortori S, Faggioni L, Cioni R, Bartolozzi C. Osteoid osteoma in atypical locations: the added value of dynamic gadolinium-enhanced MR imaging. Eur J Radiol 2009;71(03): 527-535

57 Erlemann R, Reiser M, Peters PE, Wuisman P, Niendorf HP, Kunze $\mathrm{V}$. Time-dependent changes in signal intensity in neoplastic and inflammatory lesions of the musculoskeletal system following intravenous administration of Gd-DTPA. Radiologe 1988;28(06): 269-276

58 Crespo-Rodríguez AM, De Lucas-Villarrubia JC, Pastrana-Ledesma M, Hualde-Juvera A, Méndez-Alonso S, Padron M. The diagnostic performance of non-contrast 3-Tesla magnetic resonance imaging (3-T MRI) versus 1.5-Tesla magnetic resonance arthrography (1.5-T MRA) in femoro-acetabular impingement. Eur J Radiol 2017;88:109-116

59 Chopra A, Grainger AJ, Dube B, et al. Comparative reliability and diagnostic performance of conventional $3 \mathrm{~T}$ magnetic resonance imaging and 1.5T magnetic resonance arthrography for the evaluation of internal derangement of the hip. Eur Radiol 2018; 28(03):963-971

60 Magee T. 3-T MRI of the shoulder: is MR arthrography necessary? AJR Am J Roentgenol 2009;192(01):86-92

61 Li X, Liu X, Du X, Ye Z. Diffusion-weighted MR imaging for assessing synovitis of wrist and hand in patients with rheumatoid arthritis: a feasibility study. Magn Reson Imaging 2014;32(04):350-353

62 Major NM, Browne J, Domzalski T, Cothran RL, Helms CA. Evaluation of the glenoid labrum with 3-T MRI: is intraarticular contrast necessary? AJR Am J Roentgenol 2011;196(05):1139-1144

63 Symanski JS, Subhas N, Babb J, Nicholson J, Gyftopoulos S. Diagnosis of superior labrum anterior-to-posterior tears by using MR imaging and MR arthrography: a systematic review and metaanalysis. Radiology 2017;285(01):101-113

64 Ajuied A, McGarvey CP, Harb Z, Smith CC, Houghton RP, Corbett SA. Diagnosis of glenoid labral tears using 3-tesla MRI vs. 3-tesla MRA: a systematic review and meta-analysis. Arch Orthop Trauma Surg 2018;138(05):699-709 